\title{
Overview of Beyond 4G-LTE Wireless Transmission Technologies
}

\author{
Shalini \\ M.Tech Scholar \\ Department of Electronics and Communication Engineering \\ Sagar Institute of Science \& Technology, Bhopal, India
}

\author{
Prof. Anoop Tiwari \\ Associate Professor \\ Department of Electronics and Communication Engineering \\ Sagar Institute of Science \& Technology, Bhopal, India
}

\begin{abstract}
Non-orthogonal multiple access (NOMA) is a promising applicant innovation for 5G cell systems. By and by MIMO-OFDM remote innovations are utilizing in 4G LTE development remote correspondence. This paper examine about the job of MIMO-OFDM and NOMA as principal contributing execution factors in past 4G LTE Remote Transmission Innovation from a specialized point of view. Long Term Evolution (LTE) utilizes Orthogonal Frequency Division Multiplexing (OFDM) along with MIMO (Multiple Input Multiple Output) reception apparatus innovation standard to accomplish high radio spectral efficiency and multicarrier approach for multiple accesses.
\end{abstract}

Keywords-Beyond, 4G, LTE, OFDM, MIMO, FDM.

$* * * * *$

\section{INTRODUCTION}

Long Term Evolution (LTE) is a Fourth Generation $4 \mathrm{G}$ remote broadband innovation created by the 3rd Generation Partnership Project (3GPP), an industry exchange gathering. 3GPP was built up in 1998 accordingly begun taking a shot at the radio, center system, and administration engineering of an all around pertinent Third Generation (3G) innovation determination. $3 \mathrm{G}$ is indicated by European Telecommunications Standards Institute (ETSI) and 3GPP inside the system characterized by the Worldwide Media transmission Association (ITU) standard known as Global Mobile Media transmission 2000 (ITU-200). Despite the fact that $3 \mathrm{G}$ information rates were at that point genuine in principle, introductory systems like Universal Mobile Telecommunications System (UMTS) did not promptly meet the IMT-2000 necessities in their down to earth organizations. Consequently the standards should have been improved to meet or even surpass them. The mix of High Speed Downlink Packet Access (HSDPA) and the ensuing expansion of an improved devoted channel, otherwise called High Speed Uplink Packet Access (HSUPA), prompted the advancement of the innovation alluded to as High Speed Packet Access (HSPA+) or, all the more casually, 3.5G. LTE got its name since it speaks to the following stage (4G) in a movement from GSM, a second-generation $(2 \mathrm{G})$ remote system standard, to UMTS, the third-generation 3G advancements dependent on GSM (Worldwide System for Mobile Correspondence) standard. 4G LTE gives altogether higher pinnacle information rates than the prior 3GPP innovations, The highest hypothetical information rate is 50 Mbps in uplink and with Multiple input multiple output (MIMO) the rate can be as high as $100 \mathrm{Mbps}$ in the downlink with decreased inactivity, versatile data transmission limit, short round excursion delay and in reverse similarity with existing GSM and UMTS innovation In contrast to its antecedent advances, be that as it may, LTE's upper layers use TCP/IP, empowering all traffic, for example, information, voice, video and informing to be persisted all-IP systems.

\section{A. OFDM-MIMO IN WIRELESS COMMUNICATION}

MIMO innovation has as of late risen as another worldview to accomplish exceptionally high transmission capacity efficiencies and enormous information rates in present day remote interchanges. Traditional MIMO is a cell remote innovation which empowers the utilization of multiple transmitting and getting recieving wires to move more information in less time. A MIMO direct is executed in a remote connection between $\mathrm{M}$ transmits and $\mathrm{N}$ get recieving wires. It comprises of $\mathrm{M} \times \mathrm{N}$ components that speak to the MIMO channel coefficients. NOMA is to utilize the power space for multiple access, though the past generations of mobile systems have been depending on the time/frequency/code area. Take the traditional orthogonal frequency-division multiple access (OFDMA) utilized by 3GPP-LTE for instance. A primary issue with this orthogonal multiple access (OMA) strategy is that its spectral efficiency is low when some transmission capacity assets, for example, subcarrier channels, are dispensed to clients with poor channel state data (CSI). 


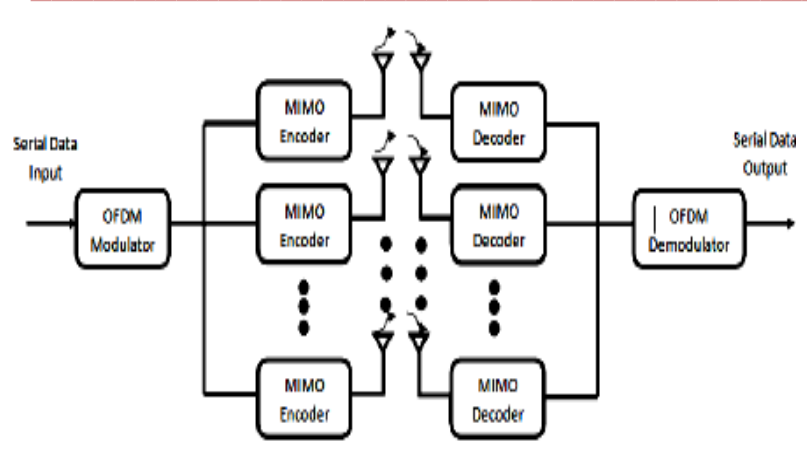

Figure 1: MIMO- OFDM in 4G LTE

Then again, the utilization of NOMA empowers every client to approach all the subcarrier channels, and thus the data transfer capacity assets allotted to the clients with poor CSI can in any case be accessed by the clients with solid CSI, which fundamentally improves the spectral efficiency. non-orthogonal multiple access (NOMA) has been as of late proposed for 3GPP Long Term Evolution (LTE) and imagined to be a fundamental segment of fifth generation (5G) mobile systems. The key component of NOMA is to serve multiple clients simultaneously/frequency/code, yet with various power levels, which yields a critical spectral efficiency increase over customary orthogonal Mama. This article gives a systematic treatment of this recently rising innovation, from its mix with multiple-input multiple-output (MIMO) advances, to helpful NOMA, just as the exchange among NOMA and subjective radio.

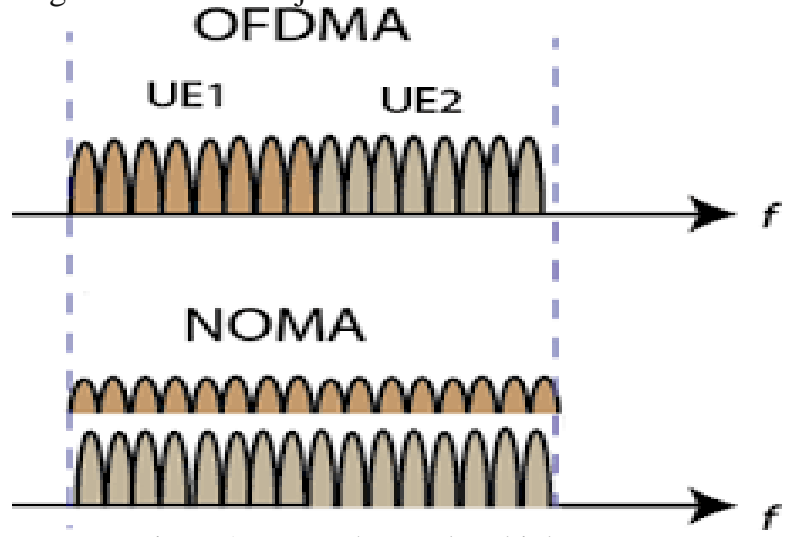

Figure 2: Non orthogonal multiple access

Presently the idea of non-orthogonal multiple access (NOMA) technique for the up and coming $5 \mathrm{G}$ systems. The majority of the current cell systems actualize orthogonal multiple access (OMA) methods, for example, time division multiple access (TDMA), frequency division multiple access (FDMA) or code division multiple access (CDMA) together. Be that as it may, none of these methods can fulfill the high needs of future radio access systems.

\section{B. NOMA in Beyond 4G LTE Communication}

The qualities of the OMA plans can be abridged as pursues. In TDMA, the data for every client is sent in non-covering availabilities, so that TDMA-based systems require exact planning synchronization, which can be testing, especially in the uplink. In FDMA usage, for example, orthogonal frequency division multiple access (OFDMA), data for every client is doled out to a subset of subcarriers. CDMA uses codes so as to isolate the clients over a similar channel. NOMA is on a very basic level not the same as these multiple access plans which give orthogonal access to the clients either in time, frequency, code or space. In NOMA, every client works in a similar band and simultaneously where they are recognized by their capacity levels. NOMA utilizes superposition coding at the transmitter with the end goal that the progressive obstruction crossing out (SIC) recipient can isolate the clients both in the uplink and in the downlink channels.

NOMA was proposed as an applicant radio access innovation for $5 \mathrm{G}$ cell systems. Reasonable usage of NOMA in cell systems requires high computational capacity to execute constant power assignment and progressive impedance retraction calculations. By 2020, the time that $5 \mathrm{G}$ systems are focused to be conveyed, the computational limit of the two handsets and access focuses is required to high enough to run NOMA algorithms.

\section{LITERATURE SURVEY}

Y. Wu et al., [1] In light of this association, this examination changes the joint advancement issue into a proportionate transfer speed streamlining issue, and further proposes an effective calculation to figure the ideal data transmission distribution (which empowers us to infer the relating transmit powers for the MUs). Broad numerical outcomes are given to approve the proposed calculation and the benefit of the proposed joint channel data transfer capacity and power allotments for the NOMA transmission.

Z. Ruoyu, et al., [2] To diminish the mind-boggling pilot overhead, a cross breed orthogonal and non-orthogonal pilot circulation at the base station (BS), which is a speculation of the current pilot dissemination conspire, is proposed by misusing the regular sparsity of channel because of the conservative radio wire plan. At that point the square sparsity for recieving wires with half breed pilot dispersion is inferred separately and can be utilized to acquire channel drive reaction.

Y. Zhang et al., [3] In the 5G remote systems, nonorthogonal multiple access (NOMA) is a promising worldview to improve its high range efficiency. This examination considers applying concurrent remote data and power move (SWIPT) strategy to agreeable NOMA remote systems, where vitality compelled transfer hubs collect the surrounding radio-frequency sign and utilize the gathered vitality to advance the packets from sources to goals.

L. You et al., [4] It is first demonstrate the shut structure eigenvectors of the ideal unicast and multicast transmit covariance frameworks, individually, which uncovers the optimality of pillar space transmission and streamlines the huge dimensional network esteemed NOUM transmission plan into a shaft area control assignment issue. Through conjuring the sunken raised strategy, we, at that point, propose a productive iterative pillar area control designation calculation with ensured combination to a stationary point.

Y. Feng et al., [5] With non-orthogonal multiple access (NOMA) in an aloof listening in situation, we handle the expansion of the mystery rate for the solid client subject to a greatest admissible mystery blackout likelihood while 
ensuring an imperative on the transmission rate to the powerless client. Just because, the reliance between the busybody's capacity to direct progressive obstruction wiping out and her channel quality is considered.

X. Wang et al., [7] In this work, consider the capability of non-orthogonal multiple access (NOMA) with the end goal of impedance moderation in downlink little cell systems (SCNs). Not quite the same as earlier works, we center around artfully multiplexing various clients on the equivalent subchannel to maintain a strategic distance from the serious between cell impedance got by ultradense organizing. Planning to augment the system throughput, we define an appropriated subchannel task issue with neighborhood data trade.

H. Zheng et al.,[8] To accomplish increasingly proficient range usage in the shrewd transportation system, nonorthogonal multiple access-empowered (NOMAempowered) V2X interchanges have been developing as a promising innovation. In this paper, the asset portion issue for NOMA-empowered V2X interchanges is examined. For V2I joins, in perspective on the client reasonableness and the various necessities of cell clients (Signals), weighted max-min rate decency for Prompts is connected, where both indistinguishable loads and various loads are considered. Concerning V2V clients (VUEs), the base sign toobstruction in addition to clamor proportion (SINR) necessities are forced on the issue detailing.

\section{OFDM Vs NOMA}

In OFDMA method BS (Base Station) shares its assets by transmitting to UEs at various time moments and frequencies. OFDMA designates subchannels and vacancies to the clients dependent on wanted transfer speed or information rate. Each of the subchannels are mapped with few number of subcarriers after stages and consequently OFDMA is strong against blurring. The advances, for example, Mobile WiMAX, LTE, LTE-progressed and 5G utilizes OFDMA strategy for asset sharing.

OFDM Utilizes the range by permitting cover. By separating the channel into narrowband level blurring subchannels, OFDM is more impervious to frequency specific blurring than single transporter systems are. Takes out ISI and IFI through utilization of a cyclic prefix. Utilizing sufficient channel coding and interleaving one can recuperate images lost because of the frequency selectivity of the channel. Channel balance ends up less difficult than by utilizing versatile balance procedures with single transporter systems. It is conceivable to utilize most extreme probability translating with sensible multifaceted nature. OFDM is computationally effective by utilizing FFT methods to execute the regulation and demodulation capacities. Is less touchy to test timing balances than single bearer systems are. Gives great security against cochannel obstruction and hasty parasitic commotion.

Impediment of OFDM is sign has a clamor like adequacy with an exceptionally enormous unique range, in this way it requires RF control intensifiers with a high crest to average power proportion. It is progressively delicate to bearer frequency balance and float than single transporter systems are because of spillage of the DFT.
The NOMA is a multiple access procedure utilized in $5 \mathrm{G}$ cell remote system. The fundamental capacity of NOMA is to serve multiple UEs (Client Types of gear) utilizing single 5G-NB (Hub B or Base Station). It serves multiple clients on same time/frequency assets. There are two fundamental methods utilized in NOMA for multiple accesses.

- Power domain: Here NOMA accomplishes multiplexing dependent on various power levels.

- Code domain: Here NOMA accomplishes multiplexing dependent on various codes.

Transmit side: NOMA utilizes superposition coding at the transmitter end. The distinctive power levels have been appointed to clients. As appeared in the figure-1, Base Station transmits superposed sign to User\#1 and User\#2. Here User\#2 utilizes high addition and User\#1 uses low increase as appeared.

Receive side: NOMA uses SIC (Progressive impedance dropping) system to recover information of both the clients. At beneficiary, User\#2 (Solid Client) substracts sign of user\#1 through SIC and later unravels its own sign. User\#1 (Frail Client) treats sign of User\#2 as commotion and deciphers its own sign straightforwardly.

Table 2: NOMA vs OFDMA

\begin{tabular}{|c|c|c|}
\hline Specifications & NOMA & OFDMA \\
\hline Full form & $\begin{array}{c}\text { Non- } \\
\text { Orthogonal } \\
\text { Multiple Access }\end{array}$ & $\begin{array}{c}\text { Orthogonal } \\
\text { Frequency } \\
\text { Division Multiple } \\
\text { Access }\end{array}$ \\
\hline $\begin{array}{c}\text { Spectrum } \\
\text { Efficiency }\end{array}$ & Higher & Lower \\
\hline $\begin{array}{c}\text { Capacity } \\
\text { (Number of } \\
\text { users/cell) }\end{array}$ & Less & More \\
\hline
\end{tabular}

\section{CONCLUSION}

In this paper, the commitment of MIMO-OFDM in 4G LTE remote advancements examined. The utilization of multiple recieving wires at the two closures of a remote connection (multiple input multiple output (MIMO) innovation) has as of late been exhibited to have the capability of accomplishing remarkable information rates in 4G-LTE. Orthogonal frequency division multiplexing (OFDM) fundamentally diminishes recipient multifaceted nature in remote broadband systems. The utilization of NOMA innovation for past $4 \mathrm{G}$ LTE remote advancements, in this way is by all accounts an appealing answer for future broadband remote systems.

\section{REFERENCE}

[1]. Y. Wu et al., "Resource optimisation for downlink nonorthogonal multiple access systems: a joint channel bandwidth and power allocations approach," in IET Communications, vol. 12, no. 19, pp. 2429-2437, 4112018.

[2]. Z. Ruoyu, Z. Honglin, Z. Jiayan and J. Shaobo, "Hybrid orthogonal and non-orthogonal pilot distribution based channel estimation in massive MIMO system," in Journal of Systems Engineering and Electronics, vol. 29, no. 5, pp. 881898, Oct. 2018. 
[3]. Y. Zhang, J. He, S. Guo and F. Wang, "Energy efficiency maximisation in wireless powered networks with cooperative non-orthogonal multiple access," in IET Communications, vol. 12, no. 18, pp. 2374-2383, 20112018.

[4]. L. You, J. Xiong, K. Li, W. Wang and X. Gao, "NonOrthogonal Unicast and Multicast Transmission for Massive MIMO With Statistical Channel State Information," in IEEE Access, vol. 6, pp. 66841-66849, 2018.

[5]. M. Alam and Q. Zhang, "Non-Orthogonal Multiple Access With Sequence Block Compressed Sensing Multiuser Detection for 5G," in IEEE Access, vol. 6, pp. 63058-63070, 2018.

[6]. Y. Feng, S. Yan and Z. Yang, "Secure Transmission to the Strong User in Non-Orthogonal Multiple Access," in IEEE Communications Letters, vol. 22, no. 12, pp. 2623-2626, Dec. 2018

[7]. X. Wang, H. Zhang, Y. Tian, C. Zhu and V. C. M. Leung, "Optimal Distributed Interference Mitigation for Small Cell Networks With Non-Orthogonal Multiple Access: A Locally Cooperative Game," in IEEE Access, vol. 6, pp. 6310763119, 2018.

[8]. H. Zheng, H. Li, S. Hou and Z. Song, "Joint Resource Allocation With Weighted Max-Min Fairness for NOMAEnabled V2X Communications," in IEEE Access, vol. 6, pp. 65449-65462, 2018

[9]. D. Wang and S. Men, "Secure Energy Efficiency for NOMA Based Cognitive Radio Networks With Nonlinear Energy Harvesting," in IEEE Access, vol. 6, pp. 62707-62716, 2018.

[10].S. Zhang, X. Xu, H. Wang, J. Peng, D. Zhang and K. Huang, "Enhancing the Physical Layer Security of Uplink NonOrthogonal Multiple Access in Cellular Internet of Things," in IEEE Access, vol. 6, pp. 58405-58417, 2018.

[11].A. Quayum, H. Minn and Y. Kakishima, "Non-Orthogonal Pilot Designs for Joint Channel Estimation and Collision Detection in Grant-Free Access Systems," in IEEE Access, vol. 6, pp. 55186-55201, 2018.

[12].X. Fu, L. Chen and J. Yang, "Non-orthogonal frequency division multiplexing based on sparse representation," in IET Communications, vol. 12, no. 16, pp. 2005-2009, 9102018. 